\title{
Electronic health records and improved nursing management of chronic obstructive pulmonary disease
}

This article was published in the following Dove Press journal:

Patient Preference and Adherence

24 March 2015

Number of times this article has been viewed

\author{
Fengping Liu' \\ Yeqing Zou' \\ Qingmei Huang ${ }^{2}$ \\ Li Zheng ${ }^{2}$ \\ Wei Wang ${ }^{2}$
}

'Yancheng Medical College, Yancheng, Jiangsu Province, People's Republic of China; ${ }^{2}$ The First Affiliated College of Medicine, Zhejiang University, Hangzhou, Zhejiang Province, People's Republic of China
Correspondence: Wei Wang The First Affiliated College of Medicine, Zhejiang University, 79 Qingchun Road, Hangzhou, Zhejiang Province 310003,

People's Republic of China

Email wangw2002@I63.com

\begin{abstract}
This paper identifies evolving trends in the diagnosis and treatment of chronic obstructive pulmonary disease (COPD), and recommends the integration of nursing strategies in COPD management via widespread implementation of electronic health records. COPD is a complex lung disease with diverse origins, both physical and behavioral, manifested in a wide range of symptoms that further increase the patient's risk for comorbidities. Early diagnosis and effective management of COPD require monitoring of a dizzying array of COPD symptoms over extended periods of time, and nurses are especially well positioned to manage potential progressions of COPD, as frontline health care providers who obtain, record, and organize patient data. Developments in medical technology greatly aid nursing management of COPD, from the deployment of spirometry as a diagnostic tool at the family practice level to newly approved treatment options, including non-nicotine pharmacotherapies that reduce the cravings associated with tobacco withdrawal. Among new medical technologies, electronic health records have proven particularly advantageous in the management of COPD, enabling providers to gather, maintain, and reference more patient data than has ever been possible before. Thus, consistent and widespread implementation of electronic health records facilitates the coordination of diverse treatment strategies, resulting in increased positive health outcomes for patients with COPD
\end{abstract}

Keywords: COPD, epidemiology, risk factors, pathology, comorbidities, exacerbations, management

\section{Introduction}

Chronic obstructive pulmonary disease (COPD), formerly known as chronic bronchitis or emphysema, is characterized by the progressive limitation of airflow, associated with an abnormal inflammatory response of the lungs toward noxious stimuli in the form of gases or particles. ${ }^{1}$ According to the Global initiative for chronic Obstructive Lung Disease (GOLD), COPD is the fourth leading cause of chronic morbidity and mortality in the USA, and is projected to rank fifth in 2020 as a worldwide burden of disease according to the World Health Organization. ${ }^{2}$ In 2001, the GOLD expert panel of health professionals from the fields of respiratory medicine, epidemiology, socioeconomics, public health, and health education compiled a consensus report based on then new information on the pathogenic mechanisms of COPD, emphasizing the need for additional study and evaluation as the initial recommendations took effect. In 2011, GOLD released a new consensus report, ie, the Global Strategy for Diagnosis, Management, and Prevention of COPD, with subsequent updates in 2013 and 2014.

GOLD's most current reports recommend assessment of COPD based on a patient's level of symptoms, future risk of exacerbations, spirometric abnormalities, and 
identification of comorbidities. The reports further recommended treatment of patients based on a quadrant system, specifying alternate treatment routes based on the number of categories in which a patient is symptomatic. The quadrant system illustrates the benefits derived from early diagnosis of COPD, since patients symptomatic in only one category may well respond to a relatively simple intervention, while patients symptomatic in two or more categories often require multiple simultaneous treatments that become increasingly invasive. Research shows that effective utilization of the quadrant system entails extensive data collection, ideally with numerous data items collected in all four categories at each and every patient visit. ${ }^{3-5}$ This accumulation of data not only aids the early diagnosis of COPD but also enables accurate assessment of the factors contributing to any given patient's disease development and progression. This assessment, in turn, aids the selection of an appropriate course of treatment, which varies significantly from one patient to the next.

This paper opens with a brief discussion of the pathology and epidemiology of COPD as a review of the many data items to be tracked for effective COPD management. Proceeding to treatment strategies illuminates the crucial role of the nurse in COPD management as the frontline health care provider responsible for obtaining and recording data. Further discussion of treatment strategies reveals a possible leadership role for nursing administration in the management of COPD, particularly in its prevention and treatment at the earliest stages. The concluding recommendation of consistent and widespread implementation of electronic health records (EHRs) is supported by a case report from Southampton City, UK, in which a 3-year audit of hospital admission records identified a subset of patients with COPD who then received targeted treatment, resulting in significantly fewer total COPD-related hospital admissions. ${ }^{6}$

\section{Evolving trends in diagnosis and assessment of COPD}

In the 1960s, the American Thoracic Society defined chronic bronchitis as a respiratory disease characterized by the presence of cough and excessive mucus production for at least 3 months in at least 2 consecutive years. ${ }^{7}$ The American Thoracic Society defined emphysema as an anatomic alteration of the lungs characterized by abnormal enlargements of the air spaces and bronchioles, with evident destructive changes in the alveolar walls. ${ }^{7}$ Over the next 50 years, clinicians and researchers together discovered that chronic bronchitis and emphysema were both manifestations of the same destructive action of behavioral and environmental risk factors on pulmonary cell tissues. ${ }^{8,9}$ Moreover, the structural alterations associated with chronic bronchitis and emphysema often originate or reinforce comorbid conditions like cardiovascular disease, certain types of cancer, and the anxiety and depression associated with decreased physical functionality. ${ }^{10}$ Today's standards indicate that health care providers should initiate a program of consistent, long-term monitoring for COPD in any patient presenting with chest symptoms. ${ }^{2}$ This ongoing assessment necessarily evaluates the patient's symptomatology and integrates the physiological data with the patient's personal and family medical history, with particular attention to the wide array of risk factors, which may support or contraindicate different treatment options. ${ }^{2}$

\section{Pathology}

The primary pathological changes seen in COPD include evident narrowing and destruction of the terminal bronchioles, sometimes recorded to $<2 \mathrm{~mm}$ in diameter. ${ }^{11}$ In healthy patients, small airways contribute little resistance to airflow. ${ }^{12}$ However, patients with COPD experience increased small airway resistance due to increased tissue volume in the walls of the small airways. ${ }^{13}$ Characteristic cellular events in the small airways of a lung with COPD include metaplasia of the goblet cells, and replacement of the respiratory Clara cells with mucus-secreting and infiltrating mononuclear cells. ${ }^{11}$ These physiological markers can be identified in patients with COPD based on spirometric analysis, which can indicate restricted airflow whether or not other symptoms are present, and for this reason, some researchers advocate implementation of spirometric analysis in family medicine practice. ${ }^{13}$ Certainly, detection of COPD by primary care providers could facilitate early detection of the disease, which often progresses unnoticed until it has reached an advanced stage.

Research validates the implementation of spirometry at the family practice level, but also calls attention to the challenges facing such implementation. ${ }^{14,15}$ In their 2014 study of 12 primary health clinics, Saad et al found that $80 \%$ of patients targeted for spirometry based on the presence of smoking or respiratory symptoms did not have COPD. ${ }^{16}$ On the other hand, $7 \%$ of smokers and $13 \%$ of respiratory patients did have the disease at the GOLD I and II levels, with the lower levels having the fewest symptoms and the greatest potential positive response to treatment. ${ }^{16}$ Moreover, the researchers noticed no difference in the type or number of respiratory symptoms in patients with COPD versus without those COPD, demonstrating the value of spirometric analysis in early detection of the disease. ${ }^{16}$ However, the researchers 
also noted that the lack of allocated time and training of health care professionals is a significant challenge that must be overcome for implementation of spirometry at the family practice level. ${ }^{16}$ Indeed, seven additional clinics were unable to participate in the study, even though they were provided with the same equipment and minimal training as the others, because of the already substantial demands on the time of the limited staff at those primary care facilities. ${ }^{16}$

\section{Epidemiology}

In 2002, COPD became the fifth most common cause of mortality worldwide. ${ }^{17}$ Statistics present a wide range of incidence rates from one country or region to another, attributable not only to genetic, environmental, and cultural differences in the populations being studied, but also to the varying definitions of COPD applied by clinicians and researchers in different countries. ${ }^{18,19}$ The following genetic, behavioral, and environmental risk factors contribute to the development of COPD, all of which require careful, longterm monitoring for disease prevention, early diagnosis, and effective treatment. ${ }^{20}$

Genetic risk factors include defects in the genes coding for microsomal epoxide hydrolase, transforming growth factor $\beta 1$, tumor necrosis factor, and $\alpha 1$-antitrypsin, a protease inhibitor responsible for protecting the lungs from the action of neutrophil elastase. ${ }^{21-25}$ While these genetic predispositions are found in only $1 \%-3 \%$ of all patients diagnosed with COPD, these predispositions, when paired with behavioral and environmental risk factors, significantly increase the risk of developing COPD. ${ }^{25,26}$

Recurrent inhalation of tobacco smoke is the most common and detrimental behavioral risk factor for the development of COPD, particularly in high-income countries. ${ }^{27}$ A 2005 study in Sweden found that $76.2 \%$ of COPD cases were due to smoking, while a similar study in Denmark found the rate of COPD caused by smoking to be $74.6 \% .{ }^{28}$ Occupational hazards resulting in exposure to dust, chemicals, vapor, and fumes are also a strong factor in the development of COPD. ${ }^{29}$ A 2012 study in the USA found that about 19.2\% of recorded COPD cases in hospitals were attributable to work-related exposures, and $31.1 \%$ of these patients were non-smokers. ${ }^{29}$

Environmental risks include exposure to the byproducts of environmentally hazardous industries, estimated to cause COPD in $35 \%$ of people in low-income to middle-income countries. ${ }^{30-35}$ Even in wealthier countries, socioeconomic factors often place patients in or near environmentally contaminated regions, increasing their risk for COPD. ${ }^{36}$
Socioeconomic risk factors for COPD also include low levels of health and hygiene education, unavailability of adequate affordable health care, and lack of access to critical resources like clean water and sanitary residence facilities. ${ }^{36}$

Demographic categories have less impact on the likelihood of an individual developing COPD, as evidenced by the widespread prevalence of COPD around the world. Worth noting, gender is no longer considered a significant determinant in the development of COPD, since recent changes in the smoking behavior of women have leveled incidence rates based on gender. ${ }^{30,31}$ Age remains the most relevant demographic risk factor for COPD. While diminished lung function is a normal occurrence, research has shown that elderly individuals with COPD have much greater impairment of lung function than elderly individuals without COPD. ${ }^{32,33}$

On the other hand, a patient's medical history greatly affects his or her risk for COPD. Past exposure to infections like bronchitis and pneumonia, especially during the early years of life, can predispose an individual to changes in the responsiveness of the airway, which may contribute to exacerbations of COPD. ${ }^{37}$ Further complicating and reinforcing the progression of COPD are comorbidities like cardiovascular disorders, metabolic or bone diseases, stroke, cancer, anemia, osteoporosis, diabetes, depression, cachexia, and even decreased cognitive functionality. 1,10,38-45

Most patients admitted to hospitals and diagnosed with COPD are expected to be readmitted within 12 months of discharge due to persistence or exacerbation of symptoms. ${ }^{46}$ Exacerbations are acute events, such as noisy breathing, increased anxiety, or chest breathing, distinguished by a worsening of respiratory symptoms well beyond the usual variations felt by the patient, which may result in a change in pharmacological treatment. ${ }^{46}$ Exacerbations require careful management because they are the primary causes of morbidity, mortality, and increased health care costs for COPD. ${ }^{2}$ Factors that contribute to the development of an exacerbated COPD episode include bacterial or viral infections of the respiratory tract or exposure to air pollution. ${ }^{2}$

\section{Challenges to the nursing strategy for management of COPD}

The preceding overview of pathology development and associated risk factors illustrates the vast complexity of COPD causes. Predictably, management strategies for COPD are equally varied and complex, combining pharmacological treatments with health education and behavioral modification strategies. Pharmacological treatments include several types of bronchodilators, corticosteroids, and pneumonia and 
influenza vaccinations; additionally, patients with hypoxemia respond well to oxygen therapy via liquid oxygen, compressed gas, or concentrators. ${ }^{47}$ Health education strategies, such as nutritional guidance and pulmonary rehabilitation through aerobic exercise, promote behavioral modifications and improve quality of life, even if they have not been found to reverse the progression of COPD. ${ }^{47}$ Psychological counseling, participation in some kind of smoking cessation program, and development of coping mechanisms further improve health outcomes. ${ }^{47}$

However, while this range of management strategies presents diverse opportunities for improved health outcomes, not all treatments are appropriate for all patients. Indeed, several of the pharmacological treatments are contraindicated for some of the common comorbidities experienced by patients with COPD. Most notably, varenicline and bupropion, the only non-nicotine first-line medications to promote smoking cessation, carry increased risks of depression and suicide. ${ }^{48}$ Conversely, certain pharmacological treatments for depression can exacerbate certain cardiovascular conditions, which could, in turn, produce an exacerbation of COPD. ${ }^{49}$ Thus, a thorough understanding of a patient's symptomatology in the context of his or her individual medical history is absolutely essential for effective treatment of the disease, since the wrong treatment could actually result in profoundly negative health outcomes.

Given this complexity in both diagnosis and treatment, the most significant challenge to nursing management of COPD is the capacity of the medical facility's human and material resources to gather, store, and access patient information. The systemic nature of this challenge suggests a leadership role for nursing administrators, whose perspective on the operational structures of their facilities can identify areas in need of improvement and redirect resources to ameliorate these weaknesses. Certainly, nursing administrators face ever more stringent budgetary demands, and shifting resources from one department to another is often fraught with unintended consequences, especially when the shift disrupts well established procedures. However, the following programs offer tremendous potential to reduce hospital admissions for COPD, freeing resources that are currently consumed by this costly disease, which could then be available to those departments that may perceive an initial loss.

Preventative measures are always cost-effective in the long run, and community-based health education programs would go a long way toward reducing the incidence of the disease. Ideally, medical facilities could partner with social welfare organizations to provide nutritional counseling, physical education, and smoking cessation programs at low or no cost, targeting populations at risk based on socioeconomic factors. Health education programs for patients who have already been diagnosed with COPD are also beneficial, particularly those that promote adherence to treatment regimens known to be effective at combating symptoms and exacerbations. ${ }^{50}$ Such programs will be most effective when they also provide patients with coping strategies to minimize the impact of side effects associated with their recommended treatments. ${ }^{50}$

Early detection of COPD would likewise produce measurably reduced costs associated with the disease. Achieving this goal will depend on the implementation of spirometric analysis at the primary health care level, as mentioned above, and will require that medical administrators address the challenges identified by Saad et al including the lack of allocated time and training of personnel. Any program seeking to establish spirometry as a diagnostic tool for family medical practitioners must provide not only the equipment but also enough training to ensure personnel feel fully competent to use the equipment in their busy clinical environments. According to an older study of COPD training in outpatient clinics, providing enhanced COPD training to medical students prior to completion of their educational program may be especially effective at applying an integrated spirometry in primary care settings. ${ }^{51}$

Spirometric analysis is only one aspect of the comprehensive preventative program necessary to identify patients with COPD as early as possible. A truly comprehensive program requires screening in each of the risk and symptom categories identified above, and the full range of screening must be conducted not just once, but at every patient visit. At the present time, this level of monitoring is practically inconceivable, and while the standard is admirable, one would be hard pressed to find a medical facility with such capability. A 2005 study of 12 facilities in two regions of the USA found that $82 \%$ of patients had at least one error of omission reported over a 13-month period, suggesting that little is known about the relative incidence of serious errors of omission compared with errors of commission. ${ }^{52}$

Fortunately, new technology could greatly facilitate the level of data collection necessary to improve widespread early diagnosis of COPD. An increasing number of medical facilities are adopting EHRs, from the research institutions that helped to develop EHRs to small family practices, which have only been able to implement this expensive technology with funding assistance from governmental agencies. ${ }^{53}$ EHRs aid in the management of diverse chronic conditions, 
including COPD and its many comorbidities. ${ }^{53}$ EHRs facilitate collection of data by providing a programmable interface that can be selected to highlight data fields critical to management of any targeted disease. ${ }^{53}$ They can even prompt the user when a particular type of testing or treatment is indicated, so that the health care professional runs less risk of committing an error of omission. ${ }^{53}$ Moreover, EHRs are also accessible to patients, who can view their own profiles and thereby increase their participation in the management of their own health. ${ }^{53}$ The EHR is already an effective platform for health education. However, with innovative programming and effective safeguards to protect confidentiality, EHRs may eventually be able to leverage lessons from social media technology to improve popular perceptions of currently unpopular health education programs, increasing patient participation in health maintenance strategies currently resisted by those most in need of them.

\section{Conclusion}

One case study from Southampton City, UK, suggests how effective, consistent, and widespread deployment of EHRs could be in the management of COPD. The British Lung Foundation developed and implemented a strategy of primary and secondary care to reduce emergency admissions of patients with acute COPD exacerbations. ${ }^{6}$ First, researchers performed a 3-year audit of hospital admission records, and found that 34 patients comprised $22 \%$ of COPD admissions over that time period. ${ }^{6}$ These patients were then targeted with interventions ranging from health education to smoking cessation and psychological counseling, and the 30-day readmission rate for these patients dropped from $13.4 \%$ to $1.9 \%{ }^{6}$

This dramatic result indicates that comprehensive data collection and analysis holds tremendous promise for the management of COPD and other chronic illnesses. EHRs are already improving health outcomes for patients with more manageable diseases, and EHRs will certainly adapt to ever more complex health conditions. As overwhelming as the implementation of EHR technology appears at first, the benefits the technology provides far exceed the perceived risk of the initial investment. Considering the burden of paper-based data collection, EHRs promise to improve quality of life outcomes for health care professionals and patients alike.

\section{Disclosure}

The authors report no conflicts of interest in this work, and are alone responsible for the content and writing of the paper.

\section{References}

1. Vijayan VK. Chronic obstructive pulmonary disease. Indian J Med Res. 2013;137:251-269.

2. Global Initiative for Chronic Obstructive Lung Disease. Global Strategy for Diagnosis, Management, and Prevention of COPD. Available from: http://www.goldcopd.org/guidelines-global-strategy-for-diagnosismanagement.html. Accessed November 30, 2014.

3. Tsai CL, Griswold SK, Clark S, Camargo CA. Factors associated with frequency of emergency room visits for COPD exacerbations. $J$ Gen Intern Med. 2007;22:799-804.

4. Slinin Y, Guo H, Li S, et al. Association of provider-patient visit frequency and patient outcomes on hemodialysis. $\mathrm{J} \mathrm{Am} \mathrm{Soc} \mathrm{Nephrol.}$ 2012;23:1560-1567.

5. Qidwai W, Rauf MUA, Sakina S, Hamid A, Ishaque S, Ashfaq T. Frequency and associated factors for care giving among elderly patients visiting a teaching hospital in Karachi, Pakistan. PLoS One. 2011;6:e25873.

6. Wilkinson T, North M, Bourne S. Reducing hospital admissions and improving the diagnosis of COPD in Southampton City: methods and results of a 12 month service improvement project. NPJ Prim Care Respir Med. 2014;24:14035.

7. American Thoracic Society. Definitions and classification of chronic bronchitis, asthma and pulmonary emphysema. Am Rev Respir Dis. 1962;85:762-767.

8. Petty TL. The history of COPD. Int J Chron Obstruct Pulmon Dis. 2006;1:3-14.

9. Chhabra SK. COPD: evolving definitions and their impact. Indian $J$ Chest Dis Allied Sci. 2009;51:135-137.

10. Boschetto P, Beghe B, Fabbri LM, Ceconi C. Link between chronic obstructive pulmonary disease and coronary artery disease: implication for clinical practice. Respirology. 2012;17:422-431.

11. Shapiro SD, Ingenito EP. The pathogenesis of chronic obstructive pulmonary disease: advances in the past 100 years. Am J Respir Cell Mol Biol. 2005;32:367-372.

12. Burgel PR. The role of small airways in obstructive airway diseases. Eur Respir Rev. 2001;20:23-33.

13. Hogg JC, Chu F, Utokaparch S, et al. The nature of small-airway obstruction in chronic obstructive pulmonary disease. $N$ Engl J Med. 2004;350:2645-2653.

14. Qaseem A, Wilt TJ, Weinberger SE, et al. Disease: a clinical practice guideline update from the American College of Physicians, American College of Chest Physicians, American Thoracic Society, and European Respiratory Society. Ann Intern Med. 2011;155:179-191.

15. Cerveri I, Accordini S, Verlato G, et al. Variations in the prevalence across countries of chronic bronchitis and smoking habits in young adults. Eur Respir J. 2001;18:85-92.

16. Saad N, Sedeno M, Metz K, Bourbeau J. Early COPD diagnosis in family medicine practice: how to implement spirometry? Int J Family Med. 2014;2014:962901.

17. Dickens C, Katon W, Blakemore A, et al. Complex interventions that reduce urgent care use in COPD: a systematic review in meta-regression. Respir Med. 2014;108:426-437.

18. Rutten van-Mölken MP, Feenstra TL. The burden of asthma and chronic obstructive pulmonary disease: data from The Netherlands. Pharmacoeconomics. 2001;19 Suppl 2:1-6.

19. Lindberg A, Jonsson AC, RonmarkE, Lundgren R, Larsson LG, LundbackB. Prevalence of chronic obstructive pulmonary disease according to BTS, ERS, GOLD and ATS criteria in relation to doctor's diagnosis, symptoms, age, gender and smoking habits. Respiration. 2005;72:471-479.

20. Chatila WM, Thomashow BM, Minai OA, Criner GJ, Make BJ. Comorbidities in chronic obstructive pulmonary disease. Proc Am Thorac Soc. 2008;5:549-555.

21. Cheng SL, Yu CJ, Chen CJ, Yang PC. Genetic polymorphism of epoxide hydrolase and glutathione S-transferase in COPD. Eur Respir J. 2004;23:818-824.

22. Celedon JC, Lange C, Raby BA, et al. The transforming growth factorbeta 1 (TGFB1) gene is associated with chronic obstructive pulmonary disease (COPD). Hum Mol Genet. 2004;13:1649-1656. 
23. Keatings VM, Cave SJ, Henry MJ, et al. A polymorphism in the tumor necrosis factor-alpha gene promoter region may predispose to a poor prognosis in COPD. Chest. 2000;118:971-975.

24. Stoller JK, Aboussouan LS. $\alpha 1$-antitrypsin deficiency. Lancet. 2005; 365:2225-2236.

25. Gooptu B, Ekeowa UI, Lomas DA. Mechanisms of emphysema in $\alpha 1$-antitrypsin deficiency: molecular and cellular insights. Eur Respir J. 2009;34:475-488.

26. Taylor SJ, Candy B, Bryar RM, et al. Effectiveness of innovations in nurse led chronic disease management for patients with chronic obstructive pulmonary disease: systematic review of evidence. BMJ. 2005;331:485

27. Eisner MD, Anthonisen N, Coultas D, et al. An official American Thoracic Society public policy statement: novel risk factors and the global burden of chronic obstructive pulmonary disease. Am J Respir Crit Care Med. 2010;182:693-718.

28. Lokke AP, Lange H, Scharling P, Fabricius, Vestbo J. Developing COPD: a 25 year follow up study of the general population. Thorax. 2006;61:935-939.

29. Hnizdo E, Sullivan PA, Bang KM, Wagner G. Association between chronic obstructive pulmonary disease and employment by industry and occupation in the US population: a study of data from the Third National Health and Nutrition Examination Survey. Am J Epidemiol. 2012;156:738-746.

30. Aryal S, Diaz-Guzman E, Mannino DM. COPD and gender differences: an update. Transl Res. 2013;162:208-218.

31. Mannino DM, Buist AS. Global burden of COPD: risk factors, prevalence, and future trends. Lancet. 2007;370:765-773.

32. Mannino DM, Homa DM, Akinbami LJ, Ford ES, SC Redd. Chronic obstructive pulmonary disease surveillance: United States, 1971-2000. Respir Care. 2002;47:1184-1199.

33. Mannino DM, Davis KJ. Lung function decline and outcomes in an elderly population. Thorax. 2006;61:472-477.

34. Halbert RJ, Natoli JL, Gano A, Badamgarav E, Buist AS, Mannino DM. Global burden of COPD: systematic review and meta-analysis. Eur Respir J. 2006;28:523-532.

35. Ran PX, Wang C, Yao WZ, et al. [Risk factors for chronic obstructive pulmonary disease in females in Chinese rural areas]. Zhonghua Nei Ke Za Zhi. 2006;45:974-979. Chinese.

36. Jemal A, Ward E, Hao Y, Thun M. Trends in the leading causes of death in the United States, 1970-2002. JAMA. 2005;294: 1255-1259.

37. Wedzicha JA. COPD exacerbations: defining their cause and preventions. Lancet. 2007;370:786-796.
38. Curkendall SM, DeLuise C, Jones JK, et al. Cardiovascular disease in patients with chronic obstructive pulmonary disease. Ann Epidemiol. 2006;16:63-70

39. De Lucas-Ramos P, Izquierdo-Alonso JL, Moro JMR, Frances JF, Lozano PV, Bellon-Cano JM. Chronic obstructive pulmonary disease as a cardiovascular risk factor: results of a case-control study. Int J Chron Obstruct Pulmon Dis. 2012;7:679-686.

40. Sin DD, Man JP, Man SF. The risk of osteoporosis in Caucasian men and women with obstructive airways disease. Am J Med. 2003;114: $10-14$.

41. Biskobing DM. COPD and osteoporosis. Chest. 2002;121:609-620.

42. Coultas DB, Edwards DW, Barnett B, Wludyka P. Predictors of depressive symptoms in patients with COPD and health impact. COPD. 2007; 4:23-28.

43. Hanania NA, Mullerova H, Locantore NW, et al. Evaluation of COPD longitudinally to identify predictive surrogate endpoints (ECLIPSE) study investigators. Determinants of depression in the ECLIPSE chronic obstructive pulmonary disease cohort. Am J Respir Crit Care Med. 2011;183:604-611.

44. Smith BJ. Chronic obstructive pulmonary disease management trials, including coordinated care, outreach nursing, and other attempted care innovations. Respir Med. 2006;2:60-63.

45. Decramer M, Janssens W. Chronic obstructive pulmonary disease and comorbidities. Lancet Respir Med. 2013;1:73-83.

46. Celli BR, Vestibo J. The EXACT-Pro: measuring exacerbations of COPD. Am J Respir Crit Care Med. 2011;183:287-288.

47. Bauldoff GS. When breathing becomes a burden: how to help patients with COPD. Am Nurs Today. 2012:7(8).

48. Chaney SE, Sheriff S. Evidence-based treatments for smoking cessation. Nurse Pract. 2012;37:24-31.

49. Yohannes AM, Alexopoulos GS. Depression and anxiety in patients with COPD. Eur Respir Rev. 2014;23:345-349.

50. McGee SR, Irby DM. Teaching in the outpatient clinic. J Gen Intern Med. 1997;12:34-40.

51. Katsenos S, Constantopoulos SH. Long-term oxygen therapy in COPD: factors affecting and ways of improving patient compliance. Pulm Med. 2011;2011:325362.

52. Hayward RA, Asch SM, Hogan MM, Hofer TP, Kerr EA. Sins of omission: getting too little medical care may be the greatest threat to patient safety. J Gen Intern Med. 2005;20:686-691.

53. Mitchell B, Begoray DL. Electronic personal health records that enhance self-management in chronic illness. Online J Issues Nurs. 2010:15(3).
Patient Preference and Adherence

\section{Publish your work in this journal}

Patient Preference and Adherence is an international, peer-reviewed, open access journal that focuses on the growing importance of patient preference and adherence throughout the therapeutic continuum. Patient satisfaction, acceptability, quality of life, compliance, persistence and their role in developing new therapeutic modalities and compounds to optimize

\section{Dovepress}

clinical outcomes for existing disease states are major areas of interest for the journal. This journal has been accepted for indexing on PubMed Central. The manuscript management system is completely online and includes a very quick and fair peer-review system, which is all easy to use. Visit http://www. dovepress.com/testimonials.php to read real quotes from published authors. 